THE INGLORIOUS YEARS 



\section{The Inglorious Years}

THE COLLAPSE OF THE

I NDUSTRIAL ORDER

AND THE RISE OF

DIGITAL SOCIETY

RN.

Daniel Cohen

Translated by Jane Marie Todd 
English translation copyright (c) 2021 by Princeton University Press

Princeton University Press is committed to the protection of copyright and the intellectual property our authors entrust to us. Copyright promotes the progress and integrity of knowledge. Thank you for supporting free speech and the global exchange of ideas by purchasing an authorized edition of this book. If you wish to reproduce or distribute any part of it in any form, please obtain permission.

Requests for permission to reproduce material from this work should be sent to permissions@press.princeton.edu

Published by Princeton University Press

41 William Street, Princeton, New Jersey o8540

6 Oxford Street, Woodstock, Oxfordshire OX20 1TR

press.princeton.edu

All Rights Reserved

Originally published as Il faut dire que les temps ont change: Chronique (fiévreuse) d'une mutation qui inquiète

(C) Editions Albin Michel-Paris 2018

Library of Congress Cataloging-in-Publication Data

Names: Cohen, Daniel, 1953-author. | Todd, Jane Marie, 1957-translator.

Title: The inglorious years : the collapse of the industrial order and the rise of digital society / Daniel Cohen ; translated by Jane Marie Todd.

Other titles: Il faut dire que les temps ont changé. English.

Description: Princeton : Princeton University Press, [2021] | "Originally published as Il faut dire que les temps ont change: Chronique (fiévreuse) d'une mutation qui inquiète (c) Editions Albin Michel-Paris 2018." | Includes bibliographical references and index.

Identifiers: LCCN 2020052903 (print) | LCCN 2020052904 (ebook) | ISBN 9780691206158 (hardback) | ISBN 9780691222264 (ebook)

Subjects: LCSH: Social change-History-21st century. | Social changeHistory-2oth century. | Information society.

Classification: LCC HM831 .C54413 2021 (print) | LCC HM831 (ebook) | $\mathrm{DDC}_{303.48 / 33-\mathrm{dc} 23}$

LC record available at https://lccn.loc.gov/2020052903

LC ebook record available at https://lccn.loc.gov/2020052904

British Library Cataloging-in-Publication Data is available

Editorial: Hannah Paul and Josh Drake

Production Editorial: Karen Carter

Jacket/Cover Design: Michel Vrana

Production: Erin Suydam

Publicity: James Schneider and Kate Farquhar-Thomson

Jacket/Cover Credit: iStock

The translation of this book has been aided by Centre National du Livre

This book has been composed in Miller

Printed on acid-free paper. $\infty$

Printed in the United States of America

$10 \begin{array}{lllllllll}9 & 8 & 7 & 6 & 5 & 4 & 3 & 2 & 1\end{array}$ 
In Memory of Philippe 

It must be said that times have changed

It's every man for himself nowadays.

-DIANE TELL, "IF I WERE A MAN"

Time was not passing, it was turning in a circle.

- GABRIEL GARCía MÁRQUEZ, A HUNDRED YEARS OF SOLITUDE 
